Caribbean Routes: Ethnographic Experiences, Theoretical Challenges, and the Production of Knowledge

\title{
PRAN WOUT LA: Expériences et dynamiques de la mobilité haïtienne
}

\author{
Mélanie Montinard ${ }^{1}$ \\ 'Universidade Federal de Rio de Janeiro, Programa de Pós-graduação em Antropologia Social, \\ Museu Nacional, Rio de Janeiro/RJ, Brasil
}

\section{Résumé}

En privilégiant, à partir d'une perspective ethnographique, les points de vue des personnes en mouvement, le présent article cherche à traiter des différentes wout (routes, en créole haïtien) que les Haïtiens ont empruntées, spécifiquement à partir du Brésil. Je propose une analyse des configurations et des dynamiques de la mobilité pour chache lavi (chercher la vie). Quels sont les sens et les usages pratiques de la catégorie wout ? Comment s'articule la circulation des individus et des familles face aux contrôles des gouvernements et des agences d'immigration ? Comment se déploient les stratégies pour que les wout des individus puissent avoir lieu en dépit des impasses physiques et symboliques ? Chache lavi non seulement crée, construit et déconstruit des relations entre les personnes, mais il implique également des tensions et des dimensions subjectives, outre des jeux permanents entre le légal et l'illégal.

Mots clés : chache lavi, mobilité, diaspora. 


\title{
PRAN WOUT LA: \\ Experiências e dinâmicas da mobilidade haitiana
}

\section{Resumo}

Ao privilegiar, a partir de uma perspectiva etnográfica, os pontos de vista das pessoas em movimento, o presente artigo busca tratar das diferentes wout (rotas, em crioulo haitiano) que foram transitadas pelos haitianos, especificamente a partir do Brasil. Proponho uma análise das configurações e as dinâmicas da mobilidade para buscar a vida (chache lavi). Quais são os significados e os usos práticos da categoria wout? Como se articulam os movimentos dos indivíduos e das famílias junto com os controles dos governos e das agências de imigração? Como as estratégias estão implantadas para que a wout dos indivíduos possa acontecer apesar dos impasses físicos e simbólicos? O chache lavi cria, constrói e desconstrói não apenas relações entre pessoas, mas também implica tensões e dimensões subjetivas, bem como jogos permanentes entre legalidades e ilegalidades.

Palavras-chave: chache lavi, mobilidade, diáspora.

\section{PRAN WOUT LA: \\ Experiences and dynamics of Haitian mobility}

\begin{abstract}
By privileging, from an ethnographic perspective, the points of view of people on the move, this article sets out to explore the various wout (routes/roads, in Haitian Creole) undertaken by Haitians, specifically from Brazil. I propose an analysis of the configurations and dynamics of mobility involved in chache lavi (pursuing life). What are the meanings and practical uses of the category wout? How is the movement of individuals and families articulated in response to the controls imposed by governments and immigration agencies? What strategies are deployed to ensure that the wout of individuals can take place despite the many physical and symbolic impasses? Chache lavi not only creates, constructs and deconstructs relations between people; it also implies subjective tensions and dimensions, as well as a constant interplay between legal and illegal.
\end{abstract}

Keywords: chache lavi, mobility, diaspora. 


\title{
PRAN WOUT LA: \\ Expériences et dynamiques de la mobilité haïtienne
}

\author{
Mélanie Montinard
}

\section{Introduction}

Cet article examine les expériences et les dynamiques de la mobilité haïtienne et analyse, à partir des récits recueillis durant ma recherche (Montinard 2019), ${ }^{1}$ les différentes wout (routes, en créole haïtien) qu'ont parcourues les Haïtiens, spécifiquement à partir du Brésil, pour chache lavi (chercher la vie), en quête de lavi miyò (une vie meilleure), ce qui fait du mot wout une catégorie native constitutive de la mobilité haïtienne.

Si, à partir de 2010, le Brésil est devenu un lieu de passage pour de nombreux migrants haïtiens qui, à l'époque, cherchaient à rejoindre la Guyane française (Joseph 2015a), ce pays est devenu partie intégrante de l'espace sociogéographique haïtien ainsi qu'un lieu de passage et de résidence, ${ }^{2}$ s'insérant dans le vaste répertoire des paysages qui composent les différentes wout empruntées par les Haïtiens pour chache lavi. Cependant, il est important de considérer la société haïtienne également à partir de la diaspora qui y est constitutive (Joseph 2015a et 2015b ; Glick-Schiller 2011), car aucun événement particulier n’a produit ou provoqué la migration, il s'agit bien d'un ensemble historique de départs et de flux migratoires. C'est en effet un enchevêtrement de différents facteurs économiques (environ 59\% de la population vivant avec un revenu inférieur à 2,41 USD par jour selon l'enquête auprès des ménages de la Banque mondiale réalisée en 2012), sociaux, culturels, politiques (les régimes autoritaires de François puis Jean-Claude Duvalier [1957-1986] et les crises politiques qui leur ont succédé - voir Zolberg \& al. 1989) et naturels (comme les inondations, les cyclones ou le tremblement de terre de janvier 2010) qui fait d'Haïti un pays qui se construit et se reconstruit au travers des départs et de la mobilité.

Les études sur l'historicité de la migration haïtienne (Anglade 1982 ; Joseph 2015a) montrent que, depuis plus d'un siècle, les Haïtiens tentent majoritairement de rejoindre les États-Unis, l'une de leurs destinations privilégiées, pour échapper aux problèmes économiques et politiques. Or, ce mouvement migratoire s'est intensifié et complexifié en raison de la récession économique au Brésil, depuis la fin de 2015, qui a directement touché les Haïtiens y vivant depuis 2010/2011, principalement ceux qui avaient bénéficié de la forte demande d'emplois due aux événements internationaux tels que la Coupe du Monde de Football en 2014 et les Jeux Olympiques en 2016. Dans ce contexte, de nouveaux projets migratoires ont vu le jour, comme la wout Miami ou pran wout la (prendre la route), expressions utilisées par les Haïtiens pour parler d'une personne qui a emprunté la route vers les États-Unis, en traversant les frontières de différents pays d'Amérique du Sud et d'Amérique Centrale pour réaliser le rêve de devenir dyaspora.

\footnotetext{
Cette recherche s'insère dans le cadre de mon doctorat en anthropologie sociale au sein du Museu Nacional de l’Université Fédérale de Rio de Janeiro (PPGAS/MN/UFRJ).

2 Les estimations relatives au nombre d'Haïtiens ayant émigré au Brésil varient, mais selon les données du Ministère de la Justice en 2019, il y aurait environ 107 ooo Haïtiens répartis principalement dans les différents États du sud du Brésil tels que Paraná, Santa Catarina, Rio Grande do Sul, São Paulo, Rio de Janeiro, Minas Gerais, entre autres.
} 
Comme je l'ai démontré dans ma recherche doctorale (Montinard 2019), pran wout la ne se réduit pas à un seul voyage du Brésil aux États-Unis, à une décision ou un itinéraire spécifique, intégrant des représentations sur des pays tels que celui del'Oncle Sam, d'Haïti, du Brésil, du Chili, du Mexique, de la République dominicaine, du Canada, parmi tant d'autres contextes. L'expression dénote une pragmatique de la mobilité et un état d'être et de devenir au sein de la diaspora haïtienne contemporaine, faisant du terme wout une catégorie native afin de comprendre les dynamiques des réseaux de mobilité:

Pran wout la est un devenir, un état de construction, un mode d'être en mouvement intégrant des dimensions physiques et symboliques, et pouvant prendre des dérivés constitutifs de la mobilité (comme ouvè wout la - « ouvrir la route », ou kite wout la - « abandonner la route », entre autres), comme une manière d'être en différents espaces et moments de la mobilité. (op. cit., 2019: 257).

Cependant, analyser la catégorie native wout à partir de ses propres termes et contextes au sein des trajectoires individuelles en perpétuel mouvement en explorant les différentes relations et les (des)équilibres des logiques de mobilité a constitué aussi bien un défi pour l'étude ethnographique qu'une richesse analytiquement fructueuse pour une théorie ethnographique de la mobilité. En effet, l'étude ethnographique s'est déroulée en de multiples lieux et en mouvement, ce qui a impliqué des défis méthodologiques singuliers, et elle n’a été possible que grâce aux multiples insertions dans les réseaux haïtiens dont je fais partie et ce de différentes façons : en tant que chercheuse, membre d'une famille haïtienne et de ses réseaux, mais aussi en tant que co-fondatrice d'une association de migrants, ${ }^{3}$ ayant une vision de juriste et d'anthropologue, ${ }^{4}$ d'ethnographe et de " native ", de femme et de mère de deux enfants haïtiens, mariée à un Haïtien, de française et d'haïtienne, de migrante vivant au Brésil, immergée et impliquée dans les réseaux haïtiens et les réseaux de discussions sur les politiques publiques migratoires. Ainsi, ma propre recherche a nécessairement une dimension d'autoanalyse ou d'auto-ethnographie, ce qui m’a obligée à considérer mes différents engagements et les multiples lieux que j'occupe et la façon dont je suis perçue par les divers agents faisant partie de l'univers analysé. En ce sens, au sein de ma propre expérience ethnographique, la dimension de " l'objectivation participative » est indissoluble de celle de la " participation observante " (Bourdieu 1991 et 2003).

Durant ma recherche de terrain entre Haïti, le Brésil et les États-Unis - où l'analyse et la compréhension des dynamiques de la mobilité haïtienne m’a été possible à partir d'une étude ethnographique aux détours des (en)jeux de la multiplicité de mes engagements (Montinard 2019: 54-65) -, j'ai pu observer les sens du mot wout, terme utilisé par les Haïtiens en mouvement ou préparant un (nouveau) départ. En effet, ce terme peut prendre des dimensions physiques et symboliques. Lorsque l'expression wout Miami est apparue, à la fin de l'année 2015, dans le quotidien des Haïtiens au Brésil qui ont pris la route des États-Unis, elle s'est insérée dans le vocabulaire de la mobilité haïtienne avec l'expression pran wout la, rendant ainsi plus floue encore la frontière entre chacune de ces dimensions. Et s'interroger sur les sens et les usages du concept natif wout, c'est renvoyer la pragmatique de la mobilité à celle de la diaspora décrite par Joseph (2015a et 2015b) ou Glick-Schiller (2011).

\footnotetext{
3 Mon mari et moi avons créé, en 2014, le projet Haiti Aqui, qui est officiellement devenu, en 2017, notre association brésilienne d'appui à l'intégration des migrants (Mawon - www.mawon.org). L'association est membre et partenaire du Comité Étatique Intersectoriel de Politique d'Attention aux Réfugiés et aux Migrants (CEIPARM), de RedeMir, de l'Organisation Internationale pour les Migrations (OIM), entre autres.

4 Avant d'obtenir mon doctorat en anthropologie sociale au PPGAS du Museu Nacional/UFRJ, je me suis formée en droit et ai obtenu deux masters en droit international comparé et en droits de l'homme à l'Université de Münster, en Allemagne.
} 


\section{Dérivés du mot wout}

En Haïti, le mot wout se conjugue principalement avec le verbe faire au futur, $m$ pral fe wout la, ann fe wout la (littéralement, je vais « faire » la route, partons sur la route), ce qui peut révéler une dimension physique de déplacement d'un point à un autre, d'un lieu à un autre, signifiant la route qu'une personne emprunte pour se rendre à un endroit désiré. Un paysan peut dire qu'il prendra la route des montagnes (fe wout mòn), une autre personne qu'elle prendra la route du sud pour dire qu'elle se rend à Baradères ( fe wout sid) et une autre encore peut dire fe wout plezi pour indiquer qu'elle va se divertir à la plage par exemple.

En outre, fe wout peut également renvoyer à une dimension de magie (maji, mistik). Comme pour appeler la population haïtienne au vaudou et à ses pratiques afin de se mobiliser, de reprendre courage et espoir en un avenir meilleur, le groupe racine RAM chantait, dans une musique du carnaval de 1997, intitulée Zanj :5 "Messieurs dames, je vais faire une route, venez avec moi ... ne regardez pas derrière vous si vous entendez tirer, suivez-moi, ne regardez pas derrière vous si vous entendez les canons tirer, suivez-moi. $»^{6}$

Pran wout la n'est non seulement pour une personne qui entreprend un voyage, planifié ou non, qui s'établit dans la durée, plus ou moins courte, entre départs, transits et arrivées, entre rester, partir et repartir, entre stratégies individuelles et collectives, et qui navigue donc entre la dimension physique et symbolique de la wout, car le terme renvoie également à une idée de privilège pour ceux qui sont sur les wout, à un processus de réussite, de construction des sens du mot diaspora, même s'ils ont souffert (pase mizè). En effet, une réussite ne se définit pas toujours par le fait d'avoir obtenu un emploi ou de bien gagner sa vie, mais peut prendre des sens différents : par exemple, lorsqu'un père fait venir sa femme et son fils pour qu'ils vivent ensemble au Brésil, quand un couple se marie ou qu'un enfant naît au Brésil, quand l'un arrive à Miami alors qu'un autre est promu à un poste de responsable au sein d'une entreprise française à Brasilia, autant de possibilités dénotant du sens purement économique d'une réussite. Dans ce dernier cas, on entendra alors davantage des expressions comme fe mouvman, vire won, brase lari a (littéralement, faire un mouvement, tourner en rond, brasser la rue), autant de concepts qui se réfèrent à l'idée de stratégies pour gagner de l'argent (fê kob), malgré le fait que tous renvoient à une image de mouvement, de se débrouiller, sans s'insérer automatiquement dans la terminologie de la mobilité.

Si pati (partir), vwayaje (voyager), ale (aller) sont des verbes conjuguant et tissant les différents sens de la mobilité haïtienne (Montinard 2019: 177-178), ils reflètent une réalité sociale dont la destination, qu'elle soit finale ou non, informe sur les représentations de la catégorie diaspora (ti dyaspora, gwo dyaspora), comme la décrit Joseph (2015b). Si pati, kite Ayiti (partir, quitter Haïti) a été l'expérience de milliers d'Haïtiens, la grande majorité répétait les mots qu'Yves m’avait dit un jour, alors que nous organisions une fête commémorative du drapeau haïtien (le 18 mai) au sein de la communauté haïtienne de Rio de Janeiro : Ayiti pap ka kite nou, li nan kè nou (Haïti ne peut nous quitter, elle reste dans nos cœurs), une phrase ponctuée d'un lourd soupir, comme si Haïti, malgré ses précarités et le manque d'opportunités, représentait, dans le quotidien des personnes vivant à l'étranger, un espace de références, d'obligations, de mémoires, de tensions où les sentiments d'appartenance se mêlent à ceux des souvenirs, d'un pays qui nous manque. Ces liens se vivent à distance et se (re)tissent sous différentes formes, lors des voyages et des visites quand cela est possible, à travers les transferts d'argent à la famille, entre autres. Cependant, le soupir d'Yves renvoyait à une ambivalence entre le fait que la personne diaspora ne quitte jamais de facto Haïti et le fait que le pays se vive au sein de la mobilité des personnes et représente un lieu que l'on doit quitter pour chache lavi, non seulement pour soi-même, mais aussi pour ceux qui y sont restés.

\footnotetext{
A écouter sur : https://www.youtube.com/watch?v=RgIuGf79JNk.

6 Lapèsòn, $m$ pral fe yon wout, ann ale avè mwen ... mèt tande tire, piga ou vire gade, ann ale avè mwen, ou mèt tande kanon, piga ou vire gade, ann ale avè mwen. 7 Il est à noter ici que le verbe migrer est totalement absent du vocabulaire créole, alors qu'il existe en français, en anglais, en portugais ou en espagnol, par exemple.
} 
Ainsi, la personne diaspora se voit obligée de pran wout la pour reconstruire une vie à l'étranger et rechercher une vie pleine et digne (Neiburg 2017 et 2019), répondant aux obligations morales de la famille et de la communauté, une réalité qui n'existe pas seulement à partir d'Haïti, mais également des pays de passage ou de résidence, comme le Brésil. Quand Pipo a annoncé qu'il allait prendre la wout Miami, il s'est justifié en disant : bagay yo pa bon mwen bò isit, $m$ gen yon pitit Ayiti (les choses ne sont pas bonnes pour moi ici, j'ai une fille en Haïti), comme si chache lavi au Brésil ne représentait alors pas (ou plus) les sens d'être diaspora. Pipo se devait de prendre une nouvelle wout avec l'idée de devoir aider sa famille restée en Haïti, selon ce qui était pi bon (meilleur) pour lui et ses proches, révélant ainsi une forme d'échec de la wout qui l'avait amené au Brésil. Les dires de Pipo étaient scandés d'expressions comme tèt chaje (la tête est remplie de problèmes), pwoblèm pap fini (les problèmes ne finiront jamais), bagay yo rèd (les choses sont difficiles), $m$ bouke chita pa fe anyen (je suis fatigué d'être assis à ne rien faire), entre autres, qui me glacent toujours, car j'avais pleine conscience du poids social que représente le fait de ne pouvoir envoyer chaque mois de l'argent à ceux qui sont restés en Haïti. Paul m'avait un jour lancé, du seuil de l'église protestante qu'il fréquentait chaque dimanche, que « les personnes en Haïti m'attendent " (moun Ayiti yo ap tann mwen), comme pour résumer qu'il avait échoué, comme s'il était mort socialement et qu'un simple numéro de transfert envoyé sur le WhatsApp de sa femme restée en Haïti pourrait en quelque sorte le faire revivre, si seulement il trouvait un emploi.

Cette situation reflète la pluralité non seulement des relations entre les individus, mais également entre eux et leur lieu de résidence ou d'origine, révélant les perspectives, les actions et les principes moraux en jeu dans les dynamiques de la mobilité et les formes à travers lesquelles elle se construit ou se déconstruit au quotidien des individus, entre proximité et distance relationnelle, émotionnelle et territoriale, où la menace des frustrations (pwoblèm, fristrasyon, konfli) demeure présente (Neiburg 2017). En ce sens, Comerford souligne que, même en cas de coexistence pacifique, il existe « une familiarité qui nous permet de tolérer des problèmes [...] qui, en d'autres circonstances, pourraient avoir de graves conséquences ", circonstance pouvant alors " changer rapidement et de manière inattendue [...], et cela demeure toujours comme une possible perspective ou menace, même dans la plus paisible et familière coexistence " (Comerford 2003: 40).

La wout Miami répond(ait) donc avant tout aux représentations d'être diaspora, de pouvoir envoyer régulièrement de l'argent à sa famille (dans ce cas, des dollars américains, une monnaie forte), de pouvoir planifier le projet de faire venir un proche ou de visiter Haïti, entre autres. Ces représentations, ce rêve de devenir diaspora, ont eu pour conséquence d'ouvrir la route (ouvè wout la) à des milliers d'Haïtiens vivant au Brésil. Publiant de belles photos sur les réseaux sociaux, ceux qui sont arrivés aux États-Unis espèrent une régularisation afin d'ouvrir d'autres routes pour leurs proches restés en Haïti ou dans le pays de passage ou de résidence.

\section{Quand pran wout la mène à Miami}

C'est un soir de décembre 2015, lors d'un churrasco d'anniversaire, dans les rues de Merk, quartier de Cidade de Deus, à Rio de Janeiro, que j’ai entendu pour la première fois les planifications de nouvelles routes à partir du Brésil. J'ai été immiscée de facto dans une conversation où Pipo expliquait à Bob, mon mari, qu'il partirait le lendemain matin pour pran wout Miami:

Bob, tu sais que je te respecte beaucoup pour ton leadership au sein des Haïtiens de Rio de Janeiro. On est des rastas, tu nous as beaucoup appris, notre $b a z^{8}$ n'existerait déjà plus si tu n'avais pas été là.

8 Bien que polysémique, le mot renvoie à un espace de sociabilité et d'appartenance où les personnes se rencontrent. Il peut être en effet associé à un gang, à un groupe de personnes qui s'y réunissent pour parler, à un groupe d'amis, à un groupe de musique ou à une association, entre autres. 
Par respect, je suis obligé de te dire quelque chose : demain matin, Étienne et moi allons à São Paulo prendre la wout Miami. On va tenter notre chance. ${ }^{9}$

Cette nouvelle m'a laissée perplexe, car je savais que très rapidement des milliers d'autres Haïtiens allaient entreprendre ce nouveau départ (vide sou wout la). En effet, les conséquences de la présence haïtienne au Brésil, considérée comme un " problème » de gouvernement (Dias \& Vieira 2019), ont impliqué l'adoption de politiques publiques spécifiques afin de pouvoir rendre gouvernable cette nouvelle population, réanimant ainsi les discussions autour de la " gouvernementalité » définie par Foucault (2004) comme:

L'ensemble constitué par les institutions, les procédures, analyses et réflexions, les calculs et les tactiques qui permettent d'exercer cette forme bien spécifique, quoique très complexe de pouvoir qui a pour cible principale la population, pour forme majeure de savoir l'économie politique, pour instrument essentiel les dispositifs de sécurité. (Foucault, cours donné au Collège de France en 1978 intitulé Sécurité, Territoire, Population, pp. 111-112, 2004).

Aussi, quelques semaines avant que Pipo ne nous annonce la nouvelle de son départ, le gouvernement brésilien venait de publier une décision au Journal Officiel pour régulariser près de quarante-quatre mille Haïtiens dont la plupart était entrés par les frontières d'Amazonie. ${ }^{10}$ Bien que les effets de la récession économique aient commencé à se faire sentir au Brésil, la majorité des Haïtiens, à la fin de l'année 2015, avait un emploi formel, situation révélée par le rapport annuel de 2016 de l’Observatoire des Migrations Internationales (Cavaltanti \& al. 2016), en partenariat avec le Ministère du Travail et de l'Emploi et l'Université de Brasilia, qui montre aussi que la population haïtienne représentait la première nationalité sur le marché du travail (se référant à un travail déclaré), devant les Portugais : les Haïtiens sont passés de 815 immigrants en 2011 à 33154 en 2015. Selon ce rapport, il a été constaté qu'en 2013, les Portugais avaient déjà été dépassés par les Haïtiens. Ceux-ci représentaient, en 2015, 26,4\% de la force de travail immigrante au Brésil. Alors que la fin de l'année 2015 présentait un bilan positif vers une certaine volonté d'intégration des nationaux d'Haïti au Brésil, dont les politiques publiques et la situation économique répondaient plutôt favorablement, de nouvelles routes se dessinaient déjà au sein des réseaux haïtiens répondant à cet imaginaire où chache lavi ne pourrait être possible que sur les terres de l'oncle Sam.

Durant sa conversation avec Bob, Pipo révélait les stratégies individuelles et collectives qu'il avait mobilisées pour préparer son départ. Même s'il venait tout juste de faire venir l'un de ses petits frères à Rio de Janeiro, il a décidé d'entreprendre la wout Miami avec ses deux colocataires et amis d'enfance, Étienne et Kenken. Les trois amis avaient négocié leur démission au sein de l'entreprise de construction civile pour laquelle ils travaillaient et avaient utilisé l'argent de leur licenciement pour organiser leur départ et financer leur wout jusqu'en Équateur. Laissant derrière lui son frère à qui il avait trouvé un travail, Pipo s'est rendu à São Paulo avec ses deux amis pour y passer quelques semaines et obtenir une nouvelle identité africaine, avant de prendre l'avion, le 7/02/2016, pour Rio Branco, dans l'état de l'Acre.

En effet, dès les premiers mois de la wout Miami (entre fin 2015 et mars/avril 2016), il fallait acheter une fausse identité d'un des pays d'Afrique (Côte d'Ivoire, République Démocratique du Congo, Sénégal, Cameroun ou Togo, entre autres) reconnus par le Haut-Commissariat des Nations Unies pour les Réfugiés comme pays dont les ressortissants pouvaient être candidats au statut de réfugié afin d'éviter les risques de déportation dans les pays traversés par la wout. Cette identité leur permettait de traverser les frontières et d'obtenir un statut

\footnotetext{
9 Baz mwen, ou konen $m$ respekte-w anpil pou tèt lidè ou ye nan mitan nou isit Rio. Rasta nou ye, ou aprann nou anpil depi nou isit, baz la tap gentan kraze si ou pat la. Pou tèt respè sa, moblije di-w li : demen maten, nap pran wout Miami, mwen mèm ak Etienne. Nou pral tante chans nou.

10 Le Brésil a répondu au « problème " des flux migratoires haïtiens par des décisions gouvernementales comme la Résolution Normative $\mathrm{n}^{\circ} 97$ ou l'ordonnance du 12/11/2015 du Ministère du Travail et de l'Emploi (Diário Oficial da União, Section 1- 13/01/2012, p. 19) qui « dispose la concession d'un visa permanent prévu aux articles 16 de la Loi $n^{\circ}$ 6.815, du 19 août 1980, pour les nationaux d'Haïti " ou l'arrêté interministériel du 12/11/2015 (section 1, p. 48) concédant la résidence à près de 44 mille personnes. Voir également Vieira (2014 et 2017).
} 
légal provisoire de " sollicitant du statut de réfugié " dans le pays par où ils transitaient, tout comme cela avait été possible au Brésil après 2010, évitant alors tout risque de déportation. Mais après mars/avril 2016, la wout Miami était connue de tous, des États où les personnes transitaient, des médias locaux et internationaux et l'identité africaine avait perdu tout son poids dans les stratégies de passage d'une frontière à une autre, certains voyageant même avec leur passeport haïtien et leur carte d'identité brésilienne. Ces couloirs parallèles où il était possible d'acheter de faux papiers révèlent donc les " fissures » ou les stratégies à partir desquelles la mobilité se construit, montrant l'importance du rôle des agences au sein des réseaux et de la mobilité haïtienne.

Les récits de Pipo ont aussi révélé les dimensions physiques et symboliques de la wout Miami, mobilisant des ressources personnelles et collectives, des stratégies, des rencontres, le hasard ou le destin, les lwa (esprits) ou les espérances en Bondye (Dieu), quand se présentaient des obstacles tels que des montagnes, où les frontières ne devenaient plus seulement physiques, mais encore symboliques. Lors de l'un de mes voyages en Floride, alors que j’allais lui rendre visite à Margate, Pipo m’a révélé les détails de son expérience au sein de la wout Miami. Une conversation qui a commencé sur un ton très sérieux:

Chacun a vécu cette wout d'une manière différente. Pour moi, c'était un trip, tu sais. Je suis un rasta, il me faut voir chaque étape de la vie d'une manière positive et croire en moi, croire au fait que je vais réussir."

Pipo répétait souvent qu'il avait dû interdire à plusieurs reprises à son frère, resté à Rio de Janeiro, d'entreprendre la wout Miami, car l'expérience était selon lui « indigne » d'un être humain. Il avait choisi de s'appuyer sur ses croyances rastafari dont la positivité allait l'accompagner à chaque étape de la wout et lui redonner forces et courage pour y affronter les obstacles les plus difficiles. Ce choix lui a valu une position de leadership tout au long de la wout : par exemple, il s'est retrouvé à la tête d'un groupe devant traverser les eaux plutôt turbulentes sur des embarcations de fortune (la lancha) et franchir des montagnes pour rejoindre le Panama.

En effet, après quelques jours de voyage en bus depuis Quito, Pipo s'est engagé, comme d'autres milliers de personnes, sur la route qui l'a conduit à Medellín en vue de se procurer un laissez-passer de 30 jours auprès des autorités migratoires colombiennes lui permettant de continuer légalement son voyage en quête du "rêve américain ". Arrivé à Turbo, une ville de la côte caraïbe sur le Golfe d'Urabá, dans le nord de la Colombie, base de départ de la lancha pour Capurgana, Pipo a remarqué une dame dont le visage lui était familier : c'était la propriétaire de la maison que sa maman avait auparavant loué, dans le centre-ville de Gonaïves - ville dans le nord d'Haïti dont il était originaire. Cette rencontre inopinée s'est traduite par un instant de retour en Haïti, où les souvenirs qu'ils ont évoqués prenaient place, et Pipo m’a raconté combien cette dame les avait aidés, sa maman, son petit frère et lui, alors que son père venait de quitter Haïti pour les États-Unis par canter, ${ }^{12}$ un récit révélant les liens de solidarité entre les deux familles.

Lors de leurs retrouvailles, ils avaient échangé des nouvelles des uns et des autres, de ceux qui étaient restés en Haïti, de ceux qui étaient décédés, de ceux qui avaient pris la wout pour le Brésil, le Chili ou les États-Unis, chacun tentant sa chance en quête de lavi miyò. Mais leur conversation est très vite revenue sur les détails de l'organisation de la wout Miami et des stratégies à déployer pour traverser le Golfe d'Urabá et parcourir les montagnes, les rivières et les broussailles pour arriver au Panama, stratégies qui s'articulaient principalement autour de la composition et du choix du groupe. Souvent, me racontait Pipo, s'insérer dans un groupe avec des enfants ou des femmes pour une traversée en mer ou à pied dans des conditions vraiment difficiles était risqué car ils ralentissaient la marche et se plaignaient davantage ; le choix et la composition

\footnotetext{
11 Chak moun viv wout sa yon jan diferan. Ou konen, pou mwen wout sa te yon trip. Se yon rasta $m$ ye, $m$ dwe wè chak etap lavi a ak yon je pozitif. $M$ dwe kwe nan tèt mwen pou m kapab rive kote m vle a.

12 Li te pran dlo me dira Pipo, expression pour dire qu'une personne s'aventure sur une wout par la mer.
} 
du groupe étaient cependant articulés sur la nécessité d'avoir la présence de femmes afin qu'elles cuisinent pour le reste du groupe qui devait être essentiellement composé de personnes fortes et capables de supporter les conditions éprouvantes du voyage.

En effet, parvenir au Panama depuis Turbo était l'étape la plus meurtrière de la wout Miami, car il fallait s'enfoncer dans l'enfer vert du bouchon du Darién, aux mains de paramilitaires et de trafiquants. Les vidéos ou les photos montrant une forêt tropicale et amazonienne dense, avec sa faune et sa flore inconnues des Haïtiens, et témoignant des conditions extrêmes de traversée, nourrissaient toujours d'intenses discussions sur les groupes de Facebook ou de WhatsApp dont je faisais partie. Cette frontière, située dans un environnement tropical hostile appelé le bouchon du Darién, est très difficilement franchissable et aucune route ne permet de passer d'un pays à l'autre - c'est d'ailleurs le seul tronçon manquant à la route panaméricaine. À partir de Turbo, la plupart des migrants traversent clandestinement la frontière panaméenne soit par voie terrestre, en marchant dans la jungle du Darién des jours durant, soit par la mer, en prenant un bateau commercial pour traverser le Golfe d'Urubá. Selon certains récits recueillis sur les réseaux sociaux, beaucoup n’ont pas résisté aux conditions extrêmes et aux risques d'une randonnée aussi traîtresse entreprise par des randonneurs non expérimentés et non équipés et où l’on rencontrait les dangers les plus effrayants (groupes criminels, animaux sauvages, maladies, inanition ou déshydratation); les plus faibles sont morts, abandonnés sur le chemin aux cris et aux pleurs de leurs proches impuissants, qui devaient continuer leur route.

Toutefois, cette rencontre a sans aucun doute marqué la décision de Pipo et de la dame de continuer leur route ensemble, comme si " Dieu m'avait envoyé cette personne me trouver " (se Bondye ki voye moun sa bay mwen), me dit Pipo. Si la dame voyait en Pipo un jeune homme débrouillard à l'esprit positif en qui elle avait confiance car elle l'avait connu enfant, Pipo voyait en elle une femme forte et courageuse (fanm vayan) qui saurait prendre soin de lui, lui faire à manger, l'aider ou le soigner en cas de besoin. Un groupe de douze personnes a alors été formé et la dame s'est chargée d'acheter tous les accessoires utiles. Il fallait penser au plus simple et au minimum pour ne pas surcharger les sacs à dos tout en garantissant la survie de tous : acheter du savon pour le linge, des aliments non périssables, des gamelles et autres ustensiles de cuisine pour faire à manger, des médicaments en cas de maladie ou d'accident, se procurer quelques couvertures au cas où les nuits seraient froides.

Pipo et ses compagnons de voyage ont accepté de prendre la tête du groupe et ont planifié le voyage en bateau pour traverser le Golfe d'Urabá, ce qui leur a coûté 20o USD par personne. Ce dangereux périple a duré environ huit heures et les a finalement obligés à finir à pied la wout jusqu'au Panama. Pipo souriait en m'expliquant que l'embarquement dans la lancha a sans doute duré aussi longtemps que la traversée, qui devait durer de 2h3o, car il fallait peser les bagages, chaque kilo en surplus devant être payé, et gare à ceux qui n'avaient pas protégé leurs affaires avec des sacs poubelle. Cependant, une fois en mer, le moteur de la lancha s'est arrêté puis est reparti après quelques minutes comme si de rien n'était - la lancha possédait trois moteurs fonctionnant l'un après l'autre par souci d'économie d'essence. Le voyage en mer avait dû inclure une escale d'une nuit sur une île pour faire le plein d'essence et se reposer. La moindre houle sur une mer pourtant relativement calme faisait décoller la barque, qui tapait très fort et tanguait comme une coque de noix au ras de l'eau, faisant sauter les passagers qui hurlaient de peur, beaucoup ne sachant pas nager, et dont la plupart avaient le mal de mer.

Arrivé à Capurgana, le groupe dirigé par Pipo s'est une nouvelle fois engagé à pied pour franchir montagnes et broussailles, un récit qu'il m’a raconté la gorge serrée, révélant que chache lavi pouvait également se mêler au sens et aux pratiques du mistik (magie, mystique) quand la wout exigeait d'affronter des conditions de voyage extrêmes. En effet, pour arriver au Panama, le groupe a dû marcher cinq jours durant dans les montagnes, traverser des rivières en crue, marcher dans la boue, dormir d'un œil dans les broussailles, attentifs aux scorpions, vipères ou autre serpent venimeux. À Capurgana, Pipo avait négocié le voyage à 250 USD par personne avec un Indien colombien qui servirait de guide. Il m’a expliqué qu'il y avait, dans cette petite ville 
colombienne, un vrai marché pour le passage des migrants sans document : " C'est un marché, ils savent que tu es étranger et ils t'abordent en te disant leur prix. Après, tu peux négocier. C'est un vrai business dans la région. ${ }^{13}$ Parfois, il fallait attendre des jours, voire des semaines, car il était plus difficile de négocier avec les passeurs qui refusaient de s'engager à pied dans les montagnes quand il y avait des opérations militaires du gouvernement de Panama pour combattre le trafic de drogues, fragilisant davantage la région du bouchon du Darién. On entendait alors dire : « la selva come » (la jungle mange) pour indiquer que la jungle peut engloutir en cas de fortes pluies, d'opérations militaires ou de présence de groupes paramilitaires et de narcotrafiquants. Une fois la négociation faite, l'Indien avait demandé à Pipo d'organiser son groupe ; l'important était d'avoir de l'eau et des bottes pour marcher en cas de pluies. Ils ont entrepris la marche derrière l'Indien qui ouvrait la route au reste du groupe avec sa machette. Le récit de Pipo révélait une marche où il était difficile de respirer du fait de la chaleur et de l'humidité, même si l'Indien avait prévu des pauses toutes les 30 minutes pour se reposer. Il était important pour les passeurs colombiens de comprendre la composition du groupe qu'ils devaient guider jusqu’à la frontière panaméenne car leur réputation pouvait être remise en cause. Parfois, Pipo riait:

Quand nous faisions une pause pour reprendre notre souffle et nous reposer, l'Indien nous demandait de prendre des photos ou de faire des vidéos avec nos téléphones portables pour montrer à nos familles que nous allions bien, qu'il nous traitait bien. Mais c'était surtout pour envoyer à ceux qui étaient encore derrière des messages en disant qu'untel était un bon type, un bon contact, et que, une fois arrivés à Capurgana, il leur fallait le demander et négocier le passage de la frontière. ${ }^{14}$

Mais Pipo m’a confié que ce raketèts les a abandonnés après quelques heures de marche - sans doute, selon lui, parce que le groupe était trop lent du fait de la présence d'enfants en bas âge -, et ils se sont retrouvés seuls, perdus, sans carte ni boussole, et sans savoir quelle direction prendre. Alors que la panique et la peur envahissaient les esprits de chacun, les enfants pleuraient sans bien comprendre ce qui se passait, les femmes hurlaient, Bible à la main, implorant Bondye (Dieu) de les sauver de cette situation et de ne pas les abandonner. Mais Pipo m'a dit que, en tant que leader du groupe, il ne pouvait laisser place aux émotions et à la démobilisation du groupe et qu'il lui fallait trouver une solution pour survivre et parvenir au Panama. Étienne et lui ont donc très vite décidé de se séparer en deux groupes, marchant en parallèle, mais à une certaine distance de sorte que l'un pouvait communiquer avec l'autre en criant.

Avant de reprendre la marche, Pipo m’a expliqué qu'il avait longtemps parlé à la dame, lui demandant de convaincre, une fois de plus, le reste du groupe à le soutenir dans cette décision et lui confiant qu'il entendait une sorte de voix, un « ti zwazo " (petit oiseau) qui lui chantait le chemin à suivre. C’est alors que Pipo m’a révélé que, la dame étant une manbo (prêtresse du vaudou), l'avait compris et avait su préparer, avec le peu de choses à portée de sa main, un rituel pour implorer la protection des ancêtres, des lwa qui les accompagnaient durant tout le voyage pour arriver sains et saufs. Pipo m’a raconté qu'elle avait ramassé quelques plantes (fey) et les avait fait bouillir dans de l'eau sur un feu qu'elle avait confectionné avec trois pierres (wòch) au pied d'un arbre (pyebwa) qu'elle avait soigneusement choisi. Il ne s'agissait pas ici simplement de fe maji (faire de la magie) en réunissant les éléments importants du vaudou comme fey, wòch ou pyebwa, mais bien de se rapprocher des lwa et de leurs demander protection pour ouvè wout la (ouvrir la route) et les aider à vaincre les obstacles qui se présentaient. En ce sens, maji et mistik ne renvoient pas au sens strict de la magie telle que définie par Richman:

\footnotetext{
13 Se yon mache. Yo konen ou se etranje, yo rive sou ou epi yo tou bay ou pri pa yo. Apre sa, ou ka negosye. Se yon biznis nan zòn nan.

14 Lè nou te konn fe yon ti pòz pou reprann souf nou, pou nou repoze, endyen an te konn mande nou fe foto oubyen fe vídeo ak telefon nou pou nou voye bay fanmi nou pou di yo nou byen, yo trete nou byen. Men bagay la te plis pou di moun ki te dèyè sou wout la fé kontak ak nèg sa lè yo rive Capurgana epi negosye avè-l paske se yon bon nèg, lap fe-w pase fwontyè $a$.

15 Les raketè occupent une place prépondérante dans les récits de mobilité, moins en raison de la possibilité d'être considérés comme des " agents illégaux » que parce qu'ils sont acteurs et facilitateurs de la mobilité des personnes sur les wout pouvant faire face à des restrictions légales ou matérielles, générant ainsi des situations d'immobilité. Catégorie native que je définirai plus précisément aux pages 16 et suivantes de cet article.
} 
Le mot maji est souvent utilisé, au sens strict du terme, pour désigner la sorcellerie et la classe de pouvoirs connue sous le nom de pwen. Symbole dominant d'une option morale et existentielle, la magie évoque ce qui est transitoire, le contrat et l'individualisme (Richman 2005: 151).

Durant cinq jours, le chant de ti zwazo guida Pipo et le groupe jusqu'au Panama, pays où certains ont passé trois semaines alors que d'autres durent y rester trois mois, selon la position du gouvernement dans la gestion de ces migrants en situation irrégulière. Quand entendre le chant d'un ti zwazo ou pratiquer un rituel prenait les sens d'une demande de protection des esprits ou des ancêtres afin d'affronter les impasses de la wout, réciter les prières évangéliques d'une Bible que certains avaient glissée dans leur sac à dos, comme objet garant de leur protection, c'était implorer Bondye de les sauver. Pran wout la s'accompagnait donc d'éléments d'une dimension collective sur les plans spirituel et transcendantal, où les rituels et les objets étaient nécessaires à la sécurité des individus tout au long de leur voyage, certains même se devant de pratiquer un tel rituel avant leur départ (Richman 2005: 152).

Arriver au Panama n'était pas chose facile et la déclaration du gouvernement panaméen du 9 mai 2016 interdisant aux Haïtiens et aux Cubains venant de Colombie d'entrer sur son territoire a compliqué la wout de milliers d'individus souhaitant traverser plus tard le pays pour se rendre aux États-Unis. Une nouvelle impasse de la wout Miami qui a poussé à inventer de nouvelles stratégies pour franchir la frontière, outre les négociations de prix.

Lorsque Pipo et Étienne sont arrivés au Panama, dans le petit village très pauvre de Yaviza, ils ont été accueillis par une communauté indigène qui n’avait que peu à leur offrir. Ils ont négocié le passage de la frontière avec les policiers pour 100 USD chacun. Puis, Pipo et Étienne ont pris un bus pour se rendre à Paso Canoas, à la frontière avec le Costa Rica où ils se sont séparés, Pipo ayant prié son ami de continuer son chemin et lui donnant le reste de ses économies (40 USD) car Étienne, fiancé à une jeune femme vivant à Portau-Prince, n'avait qu'elle pour lui assurer le financement de sa wout. Contrairement à Pipo, Étienne n'avait pas de famille proche aux États-Unis pouvant l'aider financièrement et Pipo savait combien il était difficile pour une personne de classe moyenne vivant en Haïti d'envoyer de l'argent à un proche qui se trouvait sur la wout Miami. Pipo a attendu quelques jours avant de récupérer les 50o USD que son père lui avait envoyés par transfert international, somme qui lui a permis de négocier les services d'un raketè pour atteindre la frontière du Nicaragua, à Penas Blancas, un voyage connu sous le nom de la ruta del tráfico (la route du trafic) en raison de la circulation des drogues, des marchandises illégales, voire même du trafic de personnes. Située à proximité de la côte Pacifique, Penas Blancas, d'où une mince bande de terre permet d'entrer au Nicaragua, est le seul et unique point de passage terrestre entre les deux pays dont les impasses diplomatiques imposeront à certains de revendiquer leurs droits devant les médias locaux. Le temps d'attente pouvait aller de trois à quatre mois pour certains, alors que d'autres optaient pour différentes possibilités illégales afin de traverser la frontière : à pied par la montagne (pour 1 000-1 200 USD) ${ }_{1}^{16}$ en camion ou en bus (pour 1 500-1.800 USD) ou en bateau par Los Chiles (pour goo USD), souvent au péril de leur vie.

Si certains avaient abandonné leur emploi et disposaient de l'argent de leur licenciement ou de leur aide au chômage pour acheter des billets d'avion de Rio de Janeiro à Rio Branco et financer ainsi leur wout de la frontière péruvienne à Quito, d'autres achetaient leur billet d'avion à crédit sur leur compte bancaire et d'autres encore empruntaient à des amis vivant au Brésil. Mais s'engager sur la wout a demandé dans la plupart des cas une certaine garantie que la famille vivant en diaspora était prête à financer chaque étape où le marché des transferts d'argent a alors pris son essor, surtout à Quito (Équateur), Penas Blancas (Costa Rica) et Tijuana (Mexique).

16 Voyage de 3 jours environ si le passeur accomplit honnêtement sa mission car beaucoup abandonnent le groupe à mi-chemin et les personnes se voient obligées à faire demi-tour pour payer les services d'un autre raketè. 
Aussi, le marché illégal du rakèt a alimenté les négociations entre les raketè et les personnes engagées sur la wout qui ont fait appel aux membres de leur famille vivant en diaspora et au brassage d'argent pour obtenir un document ou passer une frontière d'un pays à un autre, les prix variant selon la demande. Plus le passage d'une frontière est demandé, comme celle de Penas Blancas entre mai et septembre 2016, plus le prix demandé par les raketè augmente, pouvant atteindre 1800 USD par personne selon le moyen de transport utilisé : bateau, véhicule motorisé privé (voiture, taxi, camion, etc.) ou public (bus), ou à pied par des routes sinueuses.

En général, la wout Miami dure entre trois et six mois et coûte jusqu'à 7500 USD par personne en moyenne, selon les informations recueillies dans les longues conversations sur les groupes de WhatsApp, qui ont fortement marqué le quotidien des Haïtiens au Brésil durant le premier semestre de l'année 2016, mais également dans les récits plus personnels recueillis lors de mes séjours à Miami et en Haïti. La durée du voyage et la valeur du financement - que les individus indiquent par l'expression fanmi-m ap ede-m (ma famille va m'aider) - ont varié selon l'intensité des flux dans le temps : plus la demande était forte, plus le prix demandé par les raketè locaux augmentait et plus la durée du voyage diminuait.

Si Pipo mentionnait le Pérou comme un vye peyi, peyi lèd, pour dire que le pays n'est pas beau, peu attrayant et où il ne ferait pas bon vivre, même en transit, il définissait le Honduras et le Guatemala comme des pays où il était facile de transiter illégalement pour environ 150 USD, bien qu'il se souvienne de scènes marquées par la violence. Alors que son récit relatait avec une certaine résignation (rezinye) les tentatives de négociation d'argent contre un laissez-passer de la part des autorités locales frontalières ou les raketè, du Pérou au Guatemala, une fois arrivé sur le sol mexicain, un certain apaisement l'a envahi avant de s'engager sur une nouvelle wout, cette fois-ci d'ordre administratif, organisée entre les autorités d'immigration mexicaines et américaines.

En effet, une fois enregistré sous sa nationalité haïtienne par les services de la Police Fédérale mexicaine, ce qui lui a valu un laissez-passer de vingt jours, Pipo a entamé un nouveau voyage de quelques jours en bus jusqu'à la frontière de Tijuana/San Diego où des services humanitaires locaux étaient déployés, à la demande de l'Immigration and Customs Enforcement et des services de douane et de protection des frontières des ÉtatsUnis, pour recevoir et abriter les migrants, bracelet électronique noir à la cheville, attendant le jour de leur audience auprès du tribunal d'immigration qui statue sur leur demande d'asile ou de réfugié. Tous attendaient le formulaire I-94 qui leur permettrait d'entrer légalement aux États-Unis, pour une durée déterminée, jusqu’à la décision judiciaire finale.

Au pied du mur grillagé marquant la frontière entre Tijuana et San Diego, beaucoup ont hésité à passer la frontière lorsque le gouvernement américain a déclaré, le 22 septembre 2016, la reprise des déportations des Haïtiens en situation irrégulière sur leur territoire. ${ }^{17}$ Les messages envoyés par les autorités américaines à ces migrants et demandeurs d'asile, qu'ils laissent entrer tout en leur promettant l'expulsion, les ont plongés dans l'incertitude. Sur le point d'atteindre la fin de leur wout, après une traversée épique de la moitié du continent américain, du Brésil à la Basse-Californie, ils se tenaient au poste-frontière de San Ysidro, côté américain, où ils présentaient leur demande pour vivre aux États-Unis, au risque, hautement probable, d'être renvoyés en Haïti. Certains se sont découragés de tenter de passer la frontière et, voyant leurs espoirs s'évanouir, ont décidé de rester à Tijuana, au pied du mur, pris au piège entre mer et désert, car ils n’avaient jamais pensé à rester au Mexique, loin de la représentation qu'ils se faisaient du rêve américain, du rêve de devenir diaspora.

Comme la majorité de ceux qui se sont engagés sur la wout Miami et qui ont été entendus par les officiers des services américains d'immigration de San Diego, Pipo a passé quatre mois dans un centre de détention avant d'être libéré, en novembre 2016. Une fois sorti, il a rejoint son père, à Margate, en Floride, chez qui il vit depuis lors. Il a attendu près de six mois avant d'obtenir son permis de travail (Employment Authorization Document), un laps de temps dont il a profité pour prendre des cours d'anglais. Durant ces quelques mois, il a passé trois

17 Article du Miami Herald du 22/09/2016. 
entretiens devant un tribunal durant lesquels il lui a été demandé d'apporter des preuves de sa demande d'asile ou de sa demande de regroupement familial, des rencontres où il était représenté et accompagné par un avocat que son père avait engagé pour 500 USD. C'est en janvier 2018 que, sur décision judiciaire, sa résidence temporaire (pour deux ans) lui a été octroyée sur la base du regroupement familial. De temps à autre, Pipo aime publier des vidéos live et des photos sur Facebook s'affichant avec sa nouvelle compagne haïtiano-américaine ou sur un yacht de luxe naviguant dans la baie de Miami Beach où le konpa ${ }^{18}$ et le whisky se mêlent à la fête sur les rythmes de groupes comme T-Vice, Nu Look, Disip, entres autres, composés d'Haïtiens résidant aux États-Unis et influents sur la scène musicale haïtienne, en Haïti et à l'étranger.

Car être diaspora, ce n'est pas seulement envoyer de l'argent ou des objets à ceux qui sont restés en Haïti, réaliser un projet social ou bien organiser le voyage d'un proche, mais c'est aussi le montrer publiquement sur les réseaux sociaux, révélant alors le comportement, la valeur morale, sociale et symbolique du succès de la wout, autant de caractéristiques définissant la personne diaspora selon l'imaginaire des Haïtiens, où l'idée de gwo dyaspora (grand diaspora) ne serait possible que vivant dans des gwo peyi (pays économiquement riches), comme les États-Unis. Chache lavi ne serait donc vrai qu'en dehors des frontières terrestres d'Haïti, à l'étranger, faisant de la mobilité une ressource cultivée pour atteindre le progrès social, économique et culturel de l’individu.

\section{Retour au Bresil ou tounen anwon}

À partir d'une ethnographie des expériences de mobilité des personnes sur la wout Miami, l'intention est de détailler les différentes stratégies utilisées par les ressortissants haïtiens pour franchir les frontières de plus en plus réglementées par les différents États des Amériques. Les wout peuvent être longues, fragmentées, dangereuses, inachevées et redéfinies tout au long du processus ; les lieux de destination peuvent même être repensés. Les processus de mobilité sont alors interrompus par des régimes d'immobilité empêchant les migrants de se déplacer dans les directions souhaitées, créant de fait une immobilité involontaire (Carling 2001) qui se révèle être la réalité de plusieurs personnes qui se sont aventurées sur les wout. Cette impossibilité de poursuivre le voyage, ajoutée à la honte de rentrer dans le pays d'origine sans avoir réussi à devenir dyaspora, a conduit à un processus de reconfiguration des villes frontalières (comme Tijuana, par exemple - Montinard 2019: 211-222 ; Lebfevre 2017) et de la dynamique des migrations internes sur le continent américain. Dans ce contexte, qui a de nombreux points communs avec les réalités de la migration et du refuge de groupes et d'individus dans d'autres parties du monde et en Amérique Latine ${ }^{19}$ les actions des intermédiaires deviennent des agents fondamentaux de la mobilité. ${ }^{20}$

En effet, une fois arrivés aux États-Unis, certains, comme Pipo, partent en quête du premier emploi pour envoyer de l'argent en Haïti, d'autres travaillent durant quelques mois et tentent d'épargner assez d'argent pour financer une nouvelle wout, celle qui les amènera au Canada, mais beaucoup sont déportés vers Haïti. En effet, la déportation (ou sa menace) a dévié la wout de beaucoup, certains préférant attendre à la frontière mexicaine de Tijuana, d'autres (en prison ou libérés sous caution) attendant le jour de leur rendez-vous au Court (tribunal américain) pour se présenter devant le juge qui statue sur leur demande d'asile ou de réfugié.

\footnotetext{
18 Genre musical haïtien popularisé par le saxophoniste et guitariste Jean-Baptiste Nemours dans les années 1950.

19 Voir Bálsamo (2009) sur les récits de jeunes migrants qui ont embarqué sur des bateaux en route vers une Europe imaginaire et se sont retrouvés en Amérique du Sud ou le documentaire de Carlos Sandoval Garcia, "Casa en Tierra Ajena " (2017), qui relate les histoires et les rêves de Nicaraguayens obligés d'émigrer vers l'Amérique du Nord. Disponible sur :

https://www.youtube.com/watch?v=AkrZIumTRjI\&fbclid=IwAR377JOnVTqlIraDeT4yhbGgToljPplXwc5cbk7-E9wxv9ZlOwXhX3iMlHY

20 Les intermédiaires, figure centrale de la littérature anthropologique classique sur la migration et la mobilité, en particulier des études sur les relations politiques et économiques au niveau local, sont généralement définis comme des brokers, terme venant de l'anglais. Voir les auteurs de l'école de Manchester (Gluckman, Mitchell et Barnes 1949 ; Fallers 1955), Eric Wolf (1956) et Clifford Geertz (1960).
} 
D’autres enfin, provisoirement libérés sur le sol américain, la peur de la déportation au ventre, s'engagent, sans même attendre la décision judiciaire, sur la wout vers le nord de l'État de New York pour rentrer au Canada, pays frontalier offrant une politique d'accueil plus favorable et où les risques de déportation seraient plus faibles.

Malgré le fait que de nouvelles wout se dessinent depuis Haïti ou le Brésil, la déportation ne constitue pas seulement un malheureux événement de migration pour un individu et sa famille qui y voient un échec, mais elle a aussi de graves conséquences sur l'économie nationale, un enjeu majeur pour Haïti qui dépend essentiellement des transferts d'argent de sa diaspora. Ce point est important puisque la diaspora haïtienne comprend plus d'un million deux cent mille personnes en déplacement régulier, ${ }^{21}$ soit 11\% de la population, selon le rapport OCDE/INURED publié en 2017 sur les interactions entre politiques publiques, migrations et développement en Haïti. En outre, selon le rapport de la Banque mondiale sur les migrations et le développement, Haïti est le septième plus grand destinataire des transferts d'argent de la diaspora, lesquels occupent une place clé dans l'économie nationale. En 2017, ce montant s'élevait à deux milliards et demi de dollars américains, soit 34\% du PIB. Environ 60\% du volume total de ces transferts proviennent des États-Unis, soit un milliard et demi de dollars américains, contre cinq cent soixante-six millions de dollars américains de la République dominicaine, cent quarante-neuf millions de dollars américains de la France et cent quarante-quatre millions de dollars américains du Canada.

Il convient de noter qu'en dix ans, entre 2007 et 2016, les transferts d'argent effectués depuis les États-Unis ont augmenté de $85,6 \%$, atteignant des valeurs plus élevées, par exemple, que les transactions effectuées dans les pays d'Amérique latine et des Caraïbes, tels que la République dominicaine $(62,9 \%)$ et la Jamaïque $(14,9 \%)$. Les transferts effectués depuis les États-Unis deviennent encore plus importants si l'on considère que les montants envoyés par les personnes de la diaspora à leurs familles ont totalisé une augmentation de $51 \%$ au cours de la même période. De plus, il existe un impact local concernant l'argent de la diaspora qui est transféré : ce qui importe aux yeux des personnes recevant ces transferts, ce n'est pas tant l'argent envoyé, que l'effet que cela peut avoir sur la vie quotidienne, puisque les transferts d'argent, d'une valeur moyenne de 200 à 300 dollars américains par personne, que chaque haïtien vivant à l'étranger envoie à un parent en Haïti, représentent $60 \%$ des revenus du ménage et ravivent les espoirs d'un nouveau projet migratoire pour réaliser le rêve de devenir dyaspora.

Si les États-Unis demeurent bien la destination préférée des Haïtiens, la destination de la wout a pu changer au cours du parcours migratoire des individus qui ont pris de nouvelles wout vers le Chili, ${ }^{22}$ pays offrant plus d'opportunités pour certains. En effet, après le Brésil, le Chili est devenu la destination de milliers de jeunes Haïtiens arpentant avec foi et détermination le hall de l'aéroport de Port-au-Prince, munis d'un passeport, d'un billet et d'argent pour s'y rendre. Mais à choisir entre aller au Chili ou repartir au Brésil, l'expression lage kò (littéralement, larguer son corps, soit se laisser aller) lancée sur un groupe de WhatsApp révèle avant tout les sens de devenir diaspora à l'étranger, où les savoir-faire et savoir-être entrent dans un jeu de tensions entre savoir manier les valeurs socio-culturelles accumulées de son expérience en tant qu'Haïtien et diaspora et savoir gérer des émotions individuelles et collectives, entre sonje lakay (avoir le mal du pays) et espoirs déçus, entre attentes et frustrations (fristrasyon).

\footnotetext{
21 Ces chiffres sont cependant sous-estimés en raison de l'importance de l'immigration irrégulière : certains estiment qu'entre 1 et 2 millions d'Haïtiens vivent aux États-Unis sans document (International Crisis Group, 2007). Les données de l'U.S. Census Bureau de 2010 indiquent que cette population est concentrée, à près de $80 \%$, dans trois états principaux : Floride, New York et Massachusetts. La Floride a le pourcentage le plus élevé d’Haïtiens (48,1\%), contre 21,6\% dans l'État de New York et 8,3\% dans le Massachusetts.

22 Article du journal Folha de São Paulo du 08/05/2016.
} 
C'est le cas de Schiller qui, lors d'une visite à l'association Mawon, alors que je m'employais à lui expliquer les démarches à suivre pour obtenir la résidence au Brésil, m’a interrompu, comme pour répondre à mon air interrogatif lorsque, feuilletant son passeport, mon regard s'est détenu sur son visa de résidence chilienne valable pour un an:

Madame Mélanie, tu ne peux pas comprendre pourquoi je ne suis pas resté au Chili, comme moi je ne comprends pas pourquoi tu ne vis pas en France avec ta famille. Beaucoup comprennent pas. Mais c'est ma décision. Ce n'était pas bon pour moi là-bas (li te vinn pat bon pou mwen). Cette décision peut paraître égoïste car j’ai de la famille en Haïti qui attend que je lui envoie le numéro du transfert d'argent, mais je pouvais vraiment plus supporter les conditions de vie là-bas. Et je ne peux pas leur demander de comprendre, ils n'y vivent pas ! Je travaillais dans une plantation de citron avec d'autres dizaines d'Haïtiens. On travaillait dehors, sous la pluie, et il y avait des jours où il faisait vraiment très froid. C'est dur de travailler dans les champs. Tu travailles par tous les temps, tu portes de lourdes charges, et même si tu as un salaire de 450 USD, tu ne vis pas mieux que ça. Ça commençait à pas aller bien pour moi. Je vivais chez un ami, et pour lui aussi ça n'allait pas bien. Mon père habite à Rio de Janeiro, alors j’ai décidé de venir le rejoindre. Même si j'entends dire que le Brésil ce n'est pas mieux que le Chili, au moins, si je dois vivre dans la misère (pase mizè), autant être auprès de mes proches. C'est pour ça que je suis venu au Brésil. ${ }^{23}$

À l'instar de Schiller, selon qui ce qui est le mieux pour une personne (li pi bon mwen), elle seule peut le mesurer et l'exprimer, certains ont quitté le Chili pour se rendre au Brésil. Ceux qui avaient conservé leur carte de résidence brésilienne, quand elle était encore valide, ont pris un vol direct pour le Brésil. En septembre 2016, Arold s'est engagé sur la wout Miami, mais il a été expulsé vers Haït par les services américains d'immigration en mai 2017. Or, comme beaucoup de ceux qui ont été renvoyés vers leur terre natale, rester en Haïti était sans doute la dernière des options qu'il aurait choisie.

En effet, Arold s'était engagé sur la wout Miami sans avoir attendu de conclure les démarches pour obtenir sa résidence brésilienne ; il a quitté le Brésil avec son attestation de demande d'asile comme seul document (provisoire) d'identité au Brésil. Mais depuis le $1^{\mathrm{er}}$ janvier 2017, sur décision du gouvernement de Michel Temer, toute personne quittant le territoire avec cette seule attestation en sa possession perd automatiquement le droit à demander un statut de réfugié, ainsi que les droits en découlant (comme le permis de travail, par exemple), et ne pouvait donc plus revenir sur le sol brésilien sans visa. Une réalité que beaucoup d'Haïtiens refoulés des États-Unis ont vécu, se voyant empêchés de repran wout la pour le Brésil. Il est donc important de ne pas réduire la wout Miami à un voyage à sens unique qui aurait comme point de départ le Brésil et comme point d'arrivée les États-Unis, faisant de chache lavi une forme de tounen anwon (tourner en rond) jusqu'à trouver le havre de paix où il fait bon vivre (ap byen viv), où il serait possible de vivre une belle vie (lavi miyò).

Quand Ti Bab a su qu'Arold, son cousin, avait été renvoyé des États-Unis, il s'est vite employé à le faire revenir à Rio de Janeiro. Il m’a contactée pour que je puisse, via nos actions et nos partenariats au sein de l'association Mawon, l'aider à obtenir un visa permanent auprès de l'Ambassade du Brésil à Port-au-Prince. Mais les démarches d'obtention du visa brésilien sont très longues (en moyenne six mois, voire plus) et il était encore incertain, à l'époque, que les Haïtiens ayant obtenu la résidence au Brésil puissent y revenir sans présenter de tampon de sortie du territoire sur leur passeport. Plus d'un an plus tard, en juillet 2018, à son retour à Rio de Janeiro et alors que je l'accompagnais à la Police Fédérale pour qu'il obtienne sa résidence brésilienne,

23 Madam Mélanie, ou ka pa konprann poukisa m pat rete viv Chili, mèm jan m pa ka konprann poukisa se pa Lafrans ou al viv ak fanmi-w. Anpil moun pap konprann. Men se desizyon pa-m. Li te vinn pat bon mwen mèm. Anpil moun ka konprann li tankou yon desizyon pou tèt pa-m sèlman, paske $m$ gen fanmi Ayiti kap tann $m$ voye nimewo transfe a bay yo. Men $m$ pat ka sipòte kondsyon vi lòt bò yo mèm mèm mèm ankò. $E m$ pa ka mande moun lakay mwen konprann, yo pa viv lòt bò a ! M tap travay nan jaden sitron ak yon lòt 10 Ayisyen. Nou tap travay deyò, anba lapli. Gen dè jou li te konn fe anpil fredi. Li pa dous travay latè non. Wap travay chak jou, anba soley cho oubyen anba lapli, wap pòte chay, epi mèm si salè 450 dola, ou pa viv pi byen pase sa. Bagay yo te komanse pat bon mwen. $M$ te rete kay yon zanmi, li mèm tou te komanse gen pwoblèm. Papa-m viv Rio de Janeiro, $m$ deside vinn jwenn li. Mèm si $m$ tande Brezil pa miyò pase Chili, si se pou-m pase mizè, pito se bò kot fanmi-m. Se sak fe m vinn Brezil. 
Arold m’a raconté qu'il avait opté pour entreprendre le chimen dekoupe (littéralement, chemin découpé) par le Chili pour revenir au Brésil y (re)planifier de nouvelles wout. Or, à Rio de Janeiro, Arold n'était pas le seul à planifier de nouveaux départs, comme si, depuis le Brésil, la wout Miami était devenue un " voyage » (vwayaj) légal sur lequel s'engageaient cette fois-ci des femmes enceintes ou des familles accompagnées de leurs enfants en bas âge.

Aussi, de nouveaux départs ou retours vers le Brésil se préparaient en Haïti, réanimant les dynamiques autour de la figure du raketè. Marlène m’avait demandé de l'aider dans ses démarches pour demander un visa pour sa sœur car se posait, une nouvelle fois, une grande difficulté : celle de prendre rendez-vous sur le site de l'Organisation Internationale pour les Migrations (O.I.M.).

Si la promulgation de la RN no 97/2012 en a été un précédent, c'est l'ordonnance du 12/11/2015 disposant sur le visa d'" accueil humanitaire " des Haïtiens - qui vient s'ajouter au droit pour une épouse ou un fils, par exemple, de demander le regroupement familial - qui a crée une filière officielle d'entrée sur le territoire via l'Ambassade du Brésil à Port-au-Prince. Dans le sillage de l'obtention d'un visa permanent pour raisons humanitaires (Vieira 2017), une résolution a amené l'Ambassade brésilienne en Haïti à signer un accord avec l'O.I.M. Le Centre de Réception des Demandes de Visas pour le Brésil (B.V.A.C.), créé le 28 septembre 2015 à Tabarre, en Haïti, a alors commencé à recevoir les Haïtiens souhaitant faire une demande de visa pour raisons humanitaires. Sous l'égide de la RN nº 97/2012, puis sous l'égide de la nouvelle loi $\mathrm{n}^{\circ}$ 13.445/2017, régie par l'arrêté interministériel n ${ }^{\circ} 10$ du 6 avril 2018 (publié le 9 avril 2018, modifié une première fois par l'arrêté du 19 novembre 2018, puis une seconde fois par celui du 20 décembre 2019), cet accord entre les deux institutions s'appuie sur la résolution disposant sur la « concession du visa temporaire et de l'autorisation de résidence, à des fins d'accueil humanitaire, pour les citoyens haïtiens et pour les apatrides », et du droit à déposer une demande de regroupement familial. Le centre B.V.A.C. est dès lors devenu le passage obligé, exclusif et officiel, pour les demandes de visa auprès de la section consulaire de l'Ambassade du Brésil à Port-au-Prince.

Jusqu'en juillet 2018, l'O.I.M. recevait dans ses bureaux une cinquantaine de demandes par jour, mais elle a alors décidé, pour permettre à un public plus large d'y avoir accès et garantir le droit de chacun à faire une demande de visa pour le Brésil, que les rendez-vous seraient pris sur son site internet. Donc pour entamer les démarches relatives à la demande de visa pour le Brésil, la personne doit au préalable prendre rendez-vous sur le site de cette organisation. Beaucoup, au Brésil et en Haïti, attendaient ce jour pour pouvoir prendre rendez-vous, car quelques clics permettraient à Pauline et Paul, par exemple, de faire venir leurs deux enfants qu'ils n'avaient pas revus depuis près de trois ans. Or, un jour, en sortant de l'église, le couple avait sollicité mon aide en m'expliquant qu'il lui était presque impossible de prendre ce rendez-vous et que leurs espérances reposaient sur l'aide que j'offrais au sein de l'association Mawon et du partenariat avec l'O.I.M. en Haïti:

Madame Mélanie, imagine-toi combien c'est difficile pour nous, les Haïtiens, de prendre rendez-vous sur le site internet. Déjà, on n'a pas d'ordinateur à la maison. Et même si on en avait un, on ne sait pas comment s'en servir. Les jeunes d'aujourd'hui savent utiliser ces machines, mais nous, on est de la vieille génération. Même si on comprend qu'aujourd'hui tout se fait sur internet, ce n'est pas accessible à tout le monde. Tu dois nous aider. On est même allé dans un cybercafé, mais le site est en français et le Brésilien ne comprend pas la langue. Tu dois nous aider. Cela fait presque trois ans que nous n'avons pas vu nos enfants. Ce n'est pas facile, ils nous manquent beaucoup, tu sais. Tu dois nous aider à prendre ce rendez-vous. Bondye te le rendra. ${ }^{24}$

\footnotetext{
24 Madam Mélanie, ou imajine-w jan sa difisil pou nou Ayisyen pou-n pran randevou sa sou sit entènèt la ? Bon, deja nou pa gen odinatè lakay nou. Epi si nou te gen youn, nou pa konen kijan pou nou itilize-l. Sa se zafe jèn timoun jodia ki al lekòl ki kapab itilize machin sa yo. Men nou mèm, se granmoun nou ye. Epi mèm si nou konprann tout bagay yo fet sou entènèt jounen jodia, se pa tout moun ki ka gen akse. Ou dwe ede nou. Nou te ale nan yon cyber café, men sit la anfranse li ye, Brezilyen pa pale franse non. Ou dwe ede nou. Sa pral fe twa zan nou poko wè timoun yo. Bagay yo pa fasil, nou sonje yo anpil. Fòk ou ede nou pran randevou sa yo. Bondye ap remèt ou li.
} 
Mais tous n'avaient pas la chance d'avoir un contact (kontak) pour leur faciliter la prise de rendez-vous sur le site. Cette réalité a réanimé les dynamiques autour du raketè, éléments constitutifs de la mobilité haïtienne (Montinard 2019: 206-224). En effet, des milliers de personnes, en Haïti et au Brésil, attendaient impatiemment l'ouverture du site de l'O.I.M. pour pouvoir faire venir un proche, mais une barrière a surgi qui en a empêché plus d'un de réaliser l'opération. De fait, le système avait été complexifié pour éviter les tentatives de piratage du site et il exigeait dorénavant l'enregistrement de la personne avec son email personnel. Si j'en comprenais la démarche, je m’interrogeais davantage sur l'accessibilité de la procédure au sein de la communauté haïtienne, du moins celle vivant à Rio de Janeiro. J'imaginais mal comment un Haïtien, à l'instar de Paul et Pauline qui m’avaient confié leurs difficultés informatiques réussirait, seul, à prendre rendez-vous sur le site : il lui aurait d'abord fallu créer un email, puis se rendre sur le site de l'O.I.M.|B.V.A.C. où il devrait rechercher, sans doute pendant de longues minutes et aux prises avec un français institutionnel, le lien pour accéder à la prise de rendez-vous. Ensuite, il devrait utiliser ses connaissances de base en anglais pour s'enregistrer, puis revenir vers sa boîte e-mail afin d'y confirmer son enregistrement en cliquant sur un lien qui lui permettrait de revenir sur le site où il devrait solliciter son rendez-vous, en informant son numéro de passeport, sa date de naissance (selon le modèle américain, encore fallait-il y penser !, c'est-à-dire année/mois/jour) et choisir une date où il y aurait des rendez-vous disponibles. Quel parcours du combattant ! Ce labyrinthe de clics où il fallait maitriser plusieurs langues en a découragé plus d'un qui, se voyant alors contraint d'en déléguer la tâche à une autre personne, en espérant qu'elle puisse réussir à effectuer l'opération, a alors souvent fait appel à des raketè exigeant jusqu'à 300 USD pour leurs frais de services.

De plus, les premiers jours d'ouverture du site, les possibilités de prise de rendez-vous étaient rares. Le portail de l'O.I.M. a été confronté à des tentatives de piratage et au très grand nombre d'accès qui ralentissaient sa vitesse et entravaient la prise de rendez-vous. Cela a provoqué l'irritation de raketè qui se sont alors employés à divulguer autant de messages que de vidéos sur les réseaux sociaux, mécanismes de communication reconnus chez eux, pour dénoncer l'inaccessibilité du site. En effet, un groupe a pris l'initiative d'organiser une petite manifestation de quelques jours devant les bureaux de l'O.I.M.|B.V.A.C. aux sons des rara, élément constitutif de la revendication et de la violence en Haïti (Kivland 2017a et 2017b), comme pour faire pression (fe presyon) face au fait qu'ils ne pouvaient répondre aux demandes de leurs clients qui s'impatientaient de recevoir la confirmation du rendez-vous pour leurs proches qu'ils attendaient au Brésil, confusion (konfizyon) qui a provoqué la fermeture des bureaux de l'organisation durant quelques jours. Le mouvement organisé par les raketè s'est rapidement essoufflé, laissant place aux plus grandes manifestations que le pays a vécu pendant les premières semaines de février de 2019, bien que les revendications sur les réseaux sociaux perdurent jusqu’à aujourd'hui, révélant les frustrations (fristrasyon) qui, comme le définit Neiburg (2019), sont:

Synonymes de vie sans perspective, d'une situation sans issue, mais également d'une menace, d'une demande d'aide, d'un signal lancé à la frontière de l'explosion et du désordre (dezòd). Se dire frustré ou annoncer que les autres sont ou peuvent être frustrés sont des tentatives de stabilisation des relations dans des contextes d'interaction incertains, de relations glissantes (relasyon oubyen sityasyon glise) [...] Décrire les sentiments de frustrations, c'est également renvoyer à la fois à un diagnostic et à une recherche de réparation, une déclaration non passive, la revendication de la restitution d'une perspective de future, de lavi miyò.

Aussi, les wout empruntées aussi bien pour quitter le Brésil que pour y retourner montrent que les stratégies de franchissement des frontières, physiques, symboliques ou technologiques, ont presque toujours recours aux raketè, élément constitutif de la mobilité haïtienne, profondément ancré dans les réseaux et les pratiques culturelles. Bien que ces intermédiaires puissent renvoyer, pour certains, à des entreprises criminelles, voire violentes, parfois assimilées à celles des coyotes, facilitant l'organisation d'un voyage illégal, s'insérant alors dans les discours autour du trafic et de la traite des personnes, les ambiguïtés autour des formes que peuvent 
prendre les raketè prennent leurs sens en ce qu'ils représentent également une alternative aux procédures légales de plus en plus rigides, comme une chance d'accéder à de nouvelles wout et d'en ouvrir. Aussi, cette vision criminaliste a entravé une perception plus fine de la complexité du raketè dans les dynamiques d'(im) mobilité. Dans certains contextes par exemple, ces intermédiaires permettent la mobilité des personnes, le passage d'une frontière, l'obtention d'un document, de billets d'avion, d'informations, qu'ils agissent en tant qu'individus (kontak, $m$ konn moun, $m$ gen moun) ou en tant qu'institutions (ajans ou ajans vwayaj) (Montinard 2019: 205-210). Ce sont donc des termes cruciaux pour une meilleure compréhension de la dynamique de la mobilité haïtienne, à partir d'Haïti ou de l'étranger.

Les ambiguïtés autour de la figure des intermédiaires, et en particulier des raketè, se révèlent dans les relations complexes que les individus et les familles peuvent entretenir avec eux, qui sont parfois un ami, un voisin, un membre de la famille, un sujet moins proche indiqué par quelqu'un, un représentant d'une autorité publique ou de la société civile, entre autres. Cette multiplicité de relations entre les intermédiaires et les migrants soulève des questions de loyauté, d'obligation, de réputation et de secret, qui vont bien au-delà d'une simple relation de marché où les gens paient pour un service.

\section{Conclusion}

A partir d'une ethnographie des expériences des personnes en circulation, cet article a décrit et analysé les dynamiques de mobilité articulées en réseaux par les récits des personnes qui quittent ou ont quitté Haïti en quête d'une vie meilleure. La mobilité de ceux qui partent peut contribuer à l'immobilité de ceux qui restent et vice versa, en particulier lorsque ceux qui s'engagent sur les wout participent aux transferts d'argent pour le maintien de ceux qui restent, ou bien encore quand ceux qui restent ou vivent à l'étranger participent au financement de ceux qui partent et s'engagent sur de nouvelles wout. Explorer les wout sur lesquelles les personnes s'engagent, c'est parler de la recherche d'une vie pleine, c'est évoquer les mécanismes et les stratégies individuelles et collectives chaque fois réinventés et développés au sein de réseaux et d'espaces où créativité, espoirs et incertitudes se côtoient, créant parfois de fortes tensions et frustrations (fristrasyon). Ces stratégies et mécanismes définissent les dynamiques de la mobilité, obligeant alors à (re)penser les territoires, les relations et les personnes.

Même si les États-Unis demeurent bien la destination préférée des Haïtiens, la wout d'une personne ne se résume pas toujours à un lieu de passage ou d'arrivée et ne signifie pas pour autant l'abandon d'une route antérieure, car si certains ont quitté Rio de Janeiro pour prendre la wout Miami, ils ont fini par revenir au Brésil. De même, alors qu'entre 2010 à 2014, l'arrivée des Haïtiens au Brésil a été articulée par la wout de la Guyane Française (Joseph 2015a), les États-Unis et le Chili devinrent quelques années après les wout principales, sans que cela ne signifie pour autant la fin de la wout vers le département d'outre-mer français, bien qu'elle ait été beaucoup moins utilisée. Comme l'a montré Handerson Joseph (2017), la mobilité se construit à travers les différentes wout qu'ont empruntées les Haïtiens tout au long des deux derniers siècles, alors que d'autres sont apparues ces dernières années. Cependant, l'apparition de nouvelles wout ne démobilise pas pour autant les anciennes, car elles sont associées à la catégorie dyaspora.

James Clifford $(1992,1997)$ utilisait la notion de voyage (travelling) au sens large pour regrouper à la fois émigrations et déplacements forcés, diasporas et zones frontières, en insistant sur la notion de zones de contacts ou de borderland pour définir les frontières. Il est cependant essentiel de comprendre les pratiques et les sens des différentes wout (ou Routes ? - voir Clifford 1997) sur lesquelles se sont engagées les personnes puisque l'expression pran wout la ne signifie pas pour autant abandonner la wout antérieure, mais bien la possibilité de chache constamment lavi, faisant des wout une catégorie associée à celle de dyaspora. Comme Arold me l'a rappelé, alors qu'il se trouvait à l'aéroport de Rio de Janeiro, assis dans l'avion qui l'amenait à Saint-Domingue: 
Rappelle-toi Méla, je te l'ai déjà dit, l'Haïtien est un chercheur. Il cherchera toujours sa route. Moi, j’y vais, je m'envole ! Je dois continuer ma wout car je ne suis pas encore arrivé à destination, là où il fera bon vivre selon moi (kote kap bon mwen pou-m byen viv). ${ }^{25}$

Car il existe bien une hiérarchie entre les différentes wout, physiques ou symboliques, une hiérarchie économique et géopolitique, tant pour les personnes vivant en Haïti que pour les Haïtiens vivant à l'étranger. D'un individu à l'autre, la wout peut donc prendre des dimensions différentes et pourra être modifiée tout au long de son processus, ce qui fait que ce n'est pas une catégorie statique, associée à un territoire de façon dichotomique comme s'il existait une nette séparation entre les lieux d'origine et les lieux de destination. Le retour à un territoire connu ou d'origine est le récit de beaucoup d'Haïtiens, s'étant engagés sur des wout encombrées d'obstacles et ayant parfois été refoulés alors que d'autres sont devenus diaspora. Pran wout la est bien un devenir. Pour reprendre le sens proposé par Biehl et Locke (2017 : 6), la notion de devenir (becoming) est un processus « qui organise nos efforts individuels et collectifs, [en insistant] sur le pouvoir plastique des personnes et sur la problématique multi-réelle de vivre à côté, à travers et malgré les effets profondément contraignants des forces sociales, structurelles et matérielles, qui sont elles-mêmes plastiques ".

Aussi, une problématisation ressortant des récits ethnographiques est la relation constante des Haïtiens avec les processus de gouvernance migratoire les plus distincts en vigueur à travers le continent américain. Sur les différentes wout physiques parcourues sont (ré)inventés des itinéraires imprévus et des pratiques inhabituelles construits en réaction constante aux tentatives de régulation étatique. Ce contact avec les structures de contrôle apparaît dans le choix d'itinéraires extrêmement dangereux et coûteux, ainsi que dans l'utilisation de services de raketè, agents intermédiaires de plus en plus ancrés dans la routine migratoire de différents groupes et individus dans le monde. En effet, dans les récits ethnographiques, des régimes d'(im) mobilité apparaissent : par exemple, les centres de détention pour étrangers et les annonces d'expulsion aux États-Unis (Montinard 2019: 223-233) ; le rôle des agences internationales dans la modulation du contrôle de la population, comme celui de l'O.I.M. en Haïti (op. cit. 2019: 297-315) ; les conséquences pratiques des différentes interdictions sur la mobilité des Haïtiens en Amérique centrale ; l'action du gouvernement brésilien pour restreindre la mobilité des personnes demandant le statut de réfugié (comme la mesure adoptée le 01/01/2017 par le gouvernement de Michel Temer - op. cit. 2019: 300) ; sans mentionner un ensemble de stratégies des ressortissants haïtiens pour contrer les pratiques de contrôle engendrées par les dispositifs étatiques.

L'anthropologie de la migration transnationale a eu tendance à se concentrer sur les lieux de départ ou de destination, l'émigration et l'immigration (Abdelmalek Sayad 1977). Bien que les réseaux sociaux et les communautés diasporiques aient fait l'objet de recherches approfondies, les intermédiaires, en tant qu'agent crucial au sein des routes migratoires, ont été plus stigmatisés et dénoncés que compris. Cependant, pour reprendre le concept d'E. Wolf (1956), le raketè serait une forme de broker, d'intermédiaire entre deux cultures (cultural-broker), entre légalité et illégalité, entre mobilité et immobilité, entre le monde des contrôles étatiques et les espoirs individuels de chache lavi sur les wout. Cette image du raketè, dont les significations et les pratiques ont été mises en évidence dans ma recherche doctorale (Montinard 2019: 194-210), en tant qu'intermédiaire intégré aux projets migratoires, apparaît dans différents ouvrages historiques sur l'immigration. Si la figure de cet intermédiaire a été associée à celle d'un " homme marginal », type de personnalité qui s'est développé à travers la migration et les relations interculturelles (Parks 1928), l'importance de ces acteurs intermédiaires n'est pas nouvelle (Yannakakis, 2008, pour le Mexique) et leur rôle n'est pas toujours limité géographiquement, comme le montrent les travaux de Madeline Hsu (2000), qui, dans ses études sur le transnationalisme et la

25 Méla, sonje $m$ te di-w sa déjà. Ayisyen se yon chèchè li ye. Lap toujou chache wout li. Mwen mèm, m ale papa! Map pran vol mwen ! M dwe kontinue wout mwen paske $m$ poko rive kote $m$ vle rive a, kote kap bon mwen pou-m byen viv. 
migration entre les États-Unis et le sud de la Chine, entre 1882 et 1943, traite d'un processus ritualisé et similaire au raketè, dans lequel les immigrants chinois ont été aidés à mémoriser les détails de « faux noms de famille " afin d'entrer aux États-Unis.

Enfin, quoique sentiwon pourrait être traduit littéralement par ceinture en français, ce mot créole renvoie avant tout à l'image de tounen anwon. On dira qu'une personne ap tounen anwon (tourne en rond) pour dire qu'elle réalise un mouvement circulaire d'un point de départ à ce même point de départ, qu'elle pivote sur elle-même en faisant un cercle, un rond. Au sens figuré, le mouvement physique en soi révèle, au sein de la mobilité haïtienne, les formes que peut prendre chache lavi renvoyant à l'image d'un sentiwon, d'une wout dessinée en forme de cercle où la personne, se voyant tounen anwon, sans issue, ne pouvant progresser, se voit obligée à retourner au point de départ. Elle s'est engagée sur la wout, elle a tenté d'avancer, de progresser, d'atteindre son rêve, mais en vain. Car chache lavi, c'est aussi bien vivre (byen viv) dans le nouveau pays de résidence et cela prend donc les formes d'une recherche d'une vie meilleure, d'une quête pour réaliser son rêve de devenir un jour dyaspora, où les stratégies individuelles et collectives sont sans cesse réinventées et repensées aux détours de nouvelles wout.

Reçu le 15 septembre 2019

Accepté le 07 janvier 2020

Relecture: Alain François

\section{Bibliographie}

ANGLADE, Georges. 1982. Espace et liberté en Haïti. Montréal: ERCE \& CRC.

BÁLSAMO, P. U. 2009. Perigoso é não correr perigo. Experiências de viajantes clandestinos em navios de carga no Atlântico Sul. Tese de Doutorado, Programa de Pós-Graduação em Antropologia Social, Universidade Federal do Rio Grande do Sul.

BANQUE MONDIALE. 2017. Rapport sur les migrations et le développement. Accessible sur: http://www. worldbank.org/en/topic/labormarkets/brief/migration-and-remittances. Consulté le: 10 janvier 2020. BIEHL, João; LOCKE, Peter. 2017. Unfinished : The Anthropology of Becoming. Durham : Duke University Press. BOURDIEU, P. 1991. "Introduction à la socioanalyse". Actes de la Recherche en Sciences Sociales, 90: 3-5. . 2003. "Lobjectivation participante". Actes de la Recherche en Sciences sociales, 150(5): 43-58.

CARLING, J. 2001. Aspiration and ability in international migration Cape Verdean experiences of mobility and immobility. Thesis submitted to Department of Sociology and Human Geography, University of Oslo.

CAVALCANTI, L. ; TADEU DE OLIVEIRA, A. ; ARAUJO, D. A. 2016. "Inserção dos imigrantes no mercado de trabalho brasileiro”. Relatório Anual. OBMigra, Brasília. Disponible sur : <http:|/obmigra.mte.gov.br| index.php/relatorio-anual>. Consulté le : 13 janvier 2019.

CHARLES, Jacqueline. 22 sept. 2016. "US shifts Haiti deportation policy and gives a warming”. In : Miami Herald. Miami. Disponible sur: <http://www.miamiherald.com/news/nation-world/world/americas/haiti/ article103373227.html . Consulté le : 17 novembre 2018.

CLIFFORD, James. 1997. Routes : Travel and Translation in the Late Twentieth Century. Cambridge : Harvard University Press. 
. 1992. “Travelling Cultures”. In : L. Grossberg ; C. Nelson ; P. Treichler (dir.), Cultural Studies. New York : Routledge. pp. 96-116.

COMERFORD, J. 2003. Como uma familia: sociabilidade, territórios de parentesco e sindicalismo rural. Rio de Janeiro: Relume Dumará.

DIÁRIO OFICIAL DA UNIÃO (DOU). 12 novembre 2015, Seção 1, p. 48. Disponible sur : <https://www. jusbrasil.com.br/diarios/104076812/dou-secao-1-12-11-2015-pg-48>. Consulté le : 17 novembre 2018. DIAS, G. M. ; VIEIRA, R. 2019. "Os limites da apropriação de um léxico migratório internacional no Brasil. Dilemas". Rev. Estud. Conflito Controle Soc.,3: 151-172.

FALLERS, I. 1955. "The predicament of the modern African chief: an instance from Uganda". American Anthropologist, 57: 290-305.

FOUCAULT, M. 2004. Sécurité, territoire, population. Paris: Seuil.

GEERTZ, C. 1960. "The Javanese kijaji: the changing role of a cultural broker". Comparative Studies in Society and History, 2: 228-249.

GLICK-SCHILLER, Nina. 2011. "Locality, Globality and the popularization of a diasporic consciousness : Learning from the Haitian case". In : R. Jackson, Geographies of the Haitian Diaspora. New York : Routledge. p. xxi-xxix.

GLUCKMAN, M. ; MITCHELL, J.C. ; BARNES, J.A. 1949. "The village headman in British Central Africa". Africa, 19: 89-106.

HSU, Madeline. Y. 2000. Dreaming of Gold, Dreaming of Home : Transnationalism and Migration Between the United States and South China, 1882-1943. Stanford: Stanford University Press.

INTERNATIONAL CRISIS GROUP. 2007. «Construire la paix en Haïti: inclure les Haïtiens de l'étranger». Rapport Amérique latine/Caraïbes, 24.

JOSEPH, Handerson. 2015a. Diáspora: as dinâmicas da mobilidade haitiana no Brasil, no Suriname e na Guiana Francesa. Thèse de Doctorat, Museu Nacional, Université Fédérale de Rio de Janeiro. 2015b. "Diáspora. Sentidos sociais e mobilidades haitianas". Horizontes Antropológicos, 21(43): 51-78. . 2017. "A historicidade da (e)migração internacional haitiana: O Brasil como novo espaço migratório". Périplos: Revista De Estudos Sobre Migrações. Dossiê: Imigração haitiana no Brasil: Estado das Artes, 1(1): 07-26. . 2019. "Diáspora". In: F. Neiburg (org.), Conversas Etnográficas Haitianas. Rio de Janeiro: Papéis

Selvagens. pp. 229-258.

KIVLAND, Chelsey. 2017a. "Street Sovereignty : Violence, Power, and Respect Among the Haitian Baz". In : R. Maguire ; S. Freeman (dir.), Who Owns Haiti : People, Power, and Sovereignty. Gainesville : University Press of Florida. pp. 140-165. . 2017b. "Carnival Bands, Popular Politics, and the Craft of Showing the People in Haiti". In :

L. Frederik; K. Marra; C. A. Schuler (dir.), Showing Off, Showing Up: Studies of Hype, Heightened Performance, and Cultural Power. Ann Arbor : University of Michigan Press. pp. 174-200.

LEFEBVRE, Marie. 2017. The journey stops here : treatment of Haitians in Mexico at the United States border in Tijuana. Capstone paper submitted in partial fulfillment of the requirements for the degree (Master of arts in Latin American Studies) - Center for Latin American Studies, Stanford University, Stanford. MINISTÉRIO DO TRABALHO E EMPREGO (MTE). Resolução Normativa n. 97, 12 janvier 2012. Diário Oficial da União, 13/01/2012, Section 1, p. 19 : « dispõe sobre a concessão do visto permanente previsto no art. 16 da Lei no 6.815, de 19 de agosto de 1980, a nacionais do Haiti. ».

MONTINARD, Mélanie V. L. 2019. Pran wout la: dinâmicas da mobilidade e das redes haitianas. Thèse de Doctorat, Museu Nacional, Université Fédérale de Rio de Janeiro.

NEIBURG, Federico. 2017. “Vidas incertas. Comentários etnográficos sobre a economia real”. In : Conférence, 21/06/2017, Université de Brasilia. Mimeo. 
. 2019. "Buscando a vida, na economia e na etnografia". In: Conferência apresentada para a promoção a Professor Titular. Departamento de Antropologia do Museu Nacional da Universidade Federal do Rio de Janeiro. Mimeo.

OCDE/INURED. 2017. Interactions entre politiques publiques, migrations et développement en Haïti. Les voies de développement. Paris: Éditions OCDE. Disponible : http://dx.doi.org/10.1787/9789264278844-fr

PARKS, R.E. 1928. "Human migration and marginal man”. In: American Journal of Sociology, 33: 881-893.

RICHMANN, Karen E. 2005. Migration and voodoo. Florida : University Press of Florida.

SANDOVAL GARCIA, C. Casa en Tierra Ajena. Reportagem 2017. Disponível em: https://www.youtube.com/wat ch?v=AkrZIumTRjI\&fbclid=IwAR377JOnVTqlIraDeT4yhbGgToljPplXwc5cbk7-EgwxvgZlOwXhX3iMlHY

SANT'ANNA, Emilio ; PRADO, Avener. 08 mai 2016. "Para fugir da crise, haitianos trocam o Brasil pelo Chile”. In: Folha de São Paulo, São Paulo. Disponible sur :

<http://www1.folha.uol.com.br/cotidiano/2016/05/1768958-para-fugir-da-crise-haitianos-trocam-o-brasilpelo-chile.shtml>. Consulté le : 17 novembre 2018.

SAYAD, A. 1977. "Les trois « âges " de l'immigration algérienne en France”. Actes de la Recherche en Sciences Sociales, juin, pp. 60-79.

VIEIRA, Rosa. 2014. Itinerâncias e governo : a mobilidade haitiana no Brasil. Dissertação (Mestrado em Sociologia e Antropologia) - Universidade Federal do Rio de Janeiro, Rio de Janeiro. . 2017. "O governo da mobilidade haitiana no Brasil". Mana. Estudos de Antropologia Social, 23(1): 229-254.

WOLF, E. 1956. "Aspects of Group Relations in a Complex Society: Mexico". American Anthropologist. 58: 10651078.

YANNAKAKIS, Yanna. 2008. The art of being in-between. Native Intermediaries, Indian Identity, and Local Rule in Colonial Oaxaca. Durham : Duke University Press.

ZOLBERG, Aristide ; SUHRKE, Astri ; AGUAYO, Sergio. 1989. Escape from violence : Conflict and the refugee crisis in the developing world. Oxford : Oxford University Press.

\section{Mélanie V. L. Montinard}

Docteure à l'Université Fédérale de Rio de Janeiro, Département d'Anthropologie Sociale, Museu Nacional, Rio de Janeiro/RJ, Brésil

Federal University of Rio de Janeiro, Post-gratuate Program in Social Anthopology, Museu Nacional, Rio de Janeiro/RJ, Brazil

https://orcid.org/0000-0002-7137-4996

Email: montinardm@gmail.com 